\title{
Monitoring the Power Requirement of Wireless Sensor Networks in Non-Homogeneous Forest Environment
}

\author{
Mukesh Kumar ${ }^{*}$, Arman Alam Ansari\#, Rohini Saxena*, A.K Jaiswal^, \\ ${ }^{\#} P G$ Student,ECE, SHIATS, Allahabad \\ *Assistant professor,ECE, SHIATS, Allahabad \\ ${ }^{\wedge}$ Head of Department ECE, SHIATS, Allahabad
}

\begin{abstract}
This paper describes the sensor nodes and its hardware constraints. This paper throws light over the energy issues of the sensor nodes while transmitting and receiving data over the distances and effects of attenuation due to several environmental parameters. This paper also gives the comparative analysis of received power at different heights of transmitter antenna.
\end{abstract}

Keywords: - Wireless sensor network (WSN), relay node (RN), sense nodes ( $S N)$, base station (BS), Transmitted power $\left(P_{t}\right)$, Received power $\left(P_{r}\right)$, analog to digital converter $(A D C)$.

\section{INTRODUCTION}

Wireless sensor network (WSN) are one of the most interesting and vast field of research in present context. WSN are new network paradigm [5] characterized by the deployment of hundreds even thousands of cost effective, energy-efficient [5], small, data- centric and application specific sensor nodes. Data-centric means retrieving information matching certain attributes rather being interested in getting data from specific node [11]. Application specific means awareness towards all the layers of specific application to perform the data aggregation and information forwarding for the intermediate nodes [11]. Each of the sense nodes collects information and route them to the base station [2]. Cooperation among the network is the prominent feature of the sensor network, where all the nodes cooperate to transfer information to reach to the users via proper transmission channel. WSN overcome the difficulties of other monitoring systems, as it requires no human presence on site [6], provide real-time interaction with activities, and maintain cost and power efficient operations.

The above features of the sensor network finds great applications in the field of health, industry, corporate offices, security, military application, agricultural field monitoring, forest monitoring i.e. For forest fire detection flood detection wild animals count etc [5]. Considering the future prospect of wireless sensor network then it is going to be integral part of our life just as the computers are in today's world. Every part of our life is going to be incorporated by their use [11].

WSN can enable long-term monitoring at scales and resolutions that are difficult. WSN can be retasked after deployment in the field based on changes in the environmental conditions of the sensor network itself, or scientific endeavour requirements, currently WSN are used in several applications related to forestry, such as soil moisture monitoring, river sensing experiments, forest fire detection, and in redwood tree observation [5]. Such applications require large-scale WSN deployment.

\section{SENSOR NODE}

Sensor nodes are very small sensing devices that sense changes in the external physical environment. Sensor nodes have four basic components:

a. A sensing sub-system for data gathering from the physical environment.

b. A processing sub-system for data processing and storage.

c. A wireless communication system for the transmission of data over the transmission channel.

d. A power source i.e. a battery which provides energy for the functioning of the sensor nodes [4].

In addition to above hardware it also consists of a location finding system, a mobilizer and a power generator [1].

Sensing sub-system constitutes two sub units sensor and analog to digital converter (ADC), the analog signals sensed by the sensor is converted to digital by ADC and then fed in to processing sub- system, processing sub-system constitutes processor and storage which manages the functions that make the sensor nodes functional with the other nodes to carry out the assigned work, and the communication system consists of, transceiver [1] which connects the sensor node to the network. The power unit has pivotal role to play which may be supported by the battery i.e. solar-cell etc. The additional hardware such as location finding system gives 
the knowledge of the location of the sensor with high accuracy which is helpful in sensor network routing and sensor task and the mobilizer is helpful in moving the sensor from one place to another.

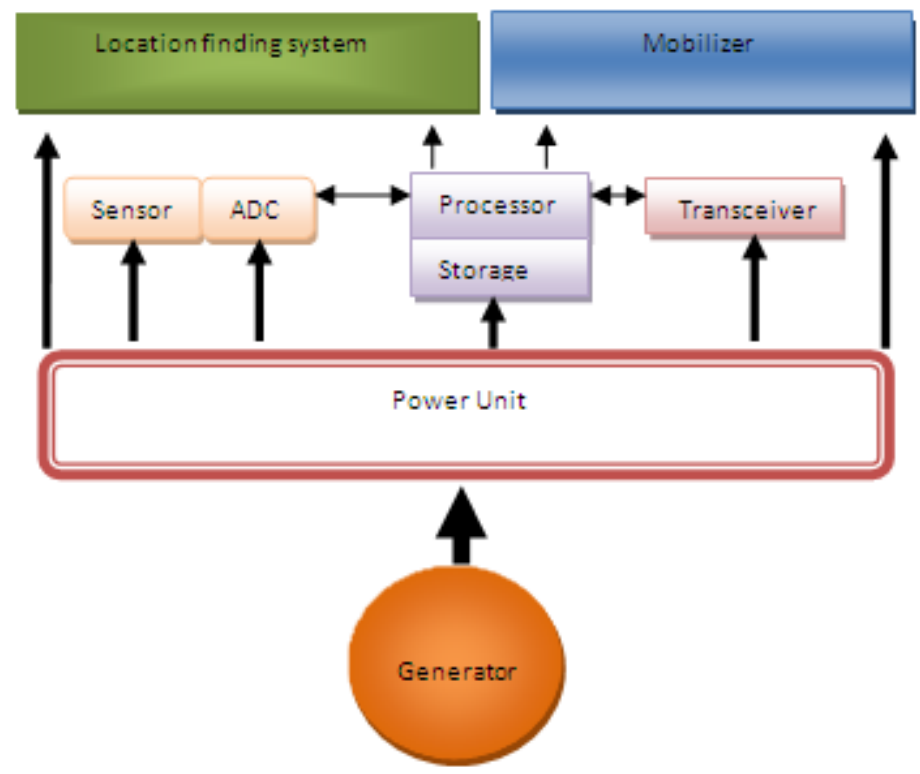

Fig: 2.1 Hardware constraints of sensor node [1]

A sensor network consists of large number of sensor nodes deployed inside the observing field or very close to it randomly [1]. The position of the sensor nodes is not needed to be pre-determined so they are allowed for their random deployment in difficult trains and in disaster relief operations. And on the other hand sensor network protocols must possess self-organising capability [3]. Another unique characteristic is the co-operative work of sensor nodes. Sensor nodes in the sensor network carry out simple calculations and transmit necessary and processed data, this feature of the sensor network puts it above over other traditional methods, and this feature improves its efficiency and accuracy.

The above features of the sensor network finds great applications in the field of health, industry, corporate offices, security, military application, agricultural field monitoring, forest monitoring i.e. For forest fire detection flood detection wild animals count etc. If we see the future prospect of wireless sensor network then it is going to be integral part of our life just as the computers are in today's world. Every part of our life is going to be incorporated by their use.

The sensor nodes gather data through their sensors process it locally or coordinate amongst neighbours and transmit the information to the user or, in general, to the data sink [7].

Since these sensor nodes have highly compact form factors, highly integrated and are wireless, they consume high energy. Furthermore, replenishing energy via replacing batteries on up to thousands of nodes (in possibly harsh terrain) is infeasible. Hence, it is necessary to conserve energy for unlocking the potential of such data gathering sensor networks so as to maximize their active lifetime after their deployment. In terms of energy consumption, the wireless exchange of data between nodes influences other node functions such as sensing and processing. Moreover, actual radios consume power not only when sending and receiving data, but also when listening. Significant energy saving can only be obtained by putting as many nodes as possible to sleep [7].

Topology management provides the distributed resources in an energy efficient manner to achieve the service requirements for the maximum possible time. Taking advantage of high-density deployment, each node can assess its connectivity and adapts its participation in the multi-hop network topology based on local measurements to increase overall system lifetime. If we increase the radio range [7] there are more nodes in the collaborative area, which can decide to go to sleep and therefore prolong the network's lifetime. So far, we have assumed that the energy required for transmission is independent of the distance. In fact, airborne radio transmissions are attenuated by a path loss with distances. Since the path loss of radio transmission scales with distance in a greater-than-linear fashion [8], the total transmission energy can be decreased by dividing a long transmission path into several shorter transmission paths. Now the problem is how we can reach the optimal range for energy efficient routing that uses the smallest amount of energy for data transmission while simultaneously allowing many nodes to be kept into the sleep state. In this paper we deduce the relationship between optimal radio ranges or distance between the nodes the transmitted power requirement, network life and the received power value in harsh forestry environment. 


\section{RECEIVED POWER [10]}

There are different parameters that affect the signal propagation in the monitored environment such as fading and shadowing. Signal attenuation, due to shadowing and fading can decrease the quality of the WSN communication. The same path with vegetation and buildings has larger attenuation than a path without any trees and buildings; hence a signal propagation model that can describe the path loss in the monitored environment is required. Signal propagation model should consider different parameters such as the heights of the building, different terrains and vegetations, transmitter antenna height and transmitter-receiver distance. As it is known that the path loss is the difference between transmitting and receiving power, and from that we conclude that the received power $\left(P_{r}\right)[10]$ in a non homogenous environment can be calculated by using equations.

$$
\begin{aligned}
& P_{r}=K_{o}-10 \gamma \log (\mathrm{d})-8.68 \alpha \mathrm{d}+\chi \\
& K_{o}=0.00169\left\{\frac{h t}{h r}\right\}^{3}+0.18269\left\{\frac{h t}{h r}\right\}^{2}-6.2337\left\{\frac{h t}{h r}\right\}+98.254 \\
& \alpha=\left(\frac{1}{1000}\right)\left\{\frac{h f}{h t(h t+h f)}\right\}
\end{aligned}
$$

Where' $\mathrm{d}$ ' is the distance between the transmitter and receiver, $\chi$ is a random variable describes the environment, $\gamma$ is the path loss exponent, $\alpha$ is the specific attenuation, $h_{t}, h_{r}$ and $h_{f}$ are the transmitter, receiver and forest or buildings mean heights, respectively. Using signal propagation model the distance ' $l$ ' between vertices in the 3D grid can be calculated, where $l$ is the minimum distance $\mathrm{d}$ that can be calculated using equation (1). Thus for the connection of two nodes the distance between them should be $\leq l$.

\section{IV.}

TRANSMITTED POWER REQUIREMENT

With the increase in the distance between the transmitting and receiving nodes placed in the nonhomogenous forestry environment there is change in the transmitting power $\left(\mathrm{P}_{\mathrm{t}}\right)$ requirement.

For a simplified power model of radio communication [9] the energy consumed per second in transmission is:

$\mathrm{P}_{\mathrm{t}}=\mathrm{B} * \mathrm{~A}\left(\mathrm{e}_{\mathrm{t}}+\mathrm{e}_{\mathrm{d}} * \mathrm{~d}^{2}\right)$

Where' $d$ ' is the distance between the transmitter and receiver, $A$ is the traffic, $B$ is the bit rate $e_{t}$ is the energy required to run the internal electronic component of sense node at transmitting end, $e_{d}$ is the amplifier consumption energy.

\section{RESULTS AND DISCUSSION}

\subsection{RECEIVED POWER $\left(P_{\mathrm{r}}\right)$ VS DISTANCE $(d)$}

In the wireless sensor networks (WSN) with the change in the distance between the nodes placed in the non-homogenous forestry environment at certain heights there is the change in the received power $\left(\mathrm{P}_{\mathrm{r}}\right)$ at the receiving end which is calculated according to the varying changing environmental parameters such as distance between the transmitter and receiver (d), random variable describing the environment $(\chi)$ which is obtained by computing the probability distribution functions (PDF) of standard data, path loss exponent $(\gamma$ ), transmitter height $\left(h_{t}\right)$, receiver height $\left(h_{r}\right)$, forest mean height $\left(h_{f}\right)$, constant depicting the relationship between the transmitting and receiving antenna heights $\left(\mathrm{K}_{0}\right)$ and the specific attenuation $(\alpha)$.

The received power decreased with the increase of distance between the transmitting and receiving nodes or in another words transmitting power requirement increases with increasing distances between the nodes.

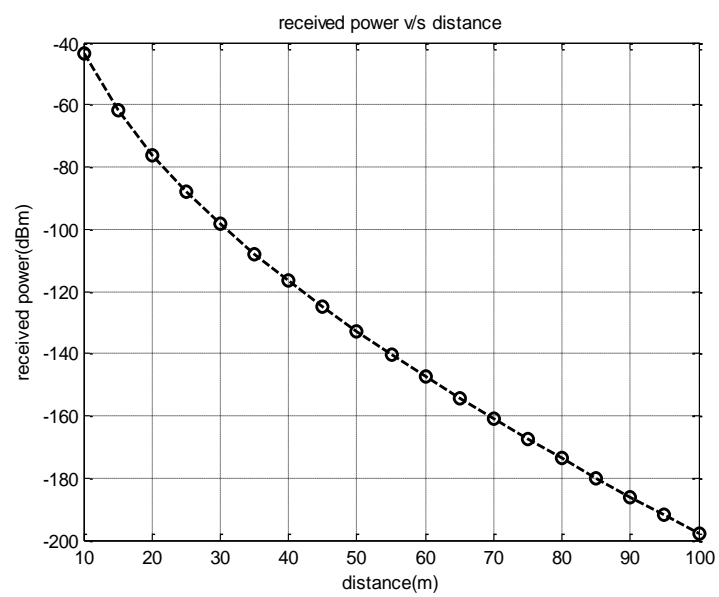

Fig 5.1 Received power Vs distance at $14 \mathrm{~m}$ transmitter height 
The Fig 5.1 depicts the variation of received power with the distance between the transmitting and receiving nodes for the different parameters for the values $h_{t}=14 \mathrm{~m}, h_{r}=1 \mathrm{~m}, h_{f}=30, \gamma=4.8, \chi=3$ and $10 \mathrm{~m} \leq d \leq 100 \mathrm{~m}$. The Fig 5.2 depicts the variation of received power with the distance between the transmitting and receiving nodes for the different parameters for the values $h_{t}=40 \mathrm{~m}, h_{r}=1 \mathrm{~m}, h_{f}=30, \gamma=3.5, \chi=3$ and $10 \mathrm{~m} \leq d \leq 100 \mathrm{~m}$.

The graph in Fig 5.3 depicts the variation of received power with the distance between the transmitting and receiving nodes for different parameters, for the values $h_{t}=70 \mathrm{~m}, h_{r}=1 \mathrm{~m}, h_{f}=30, \gamma=3.5, \chi=3$ and $10 \mathrm{~m} \leq d \leq 100 \mathrm{~m}$.

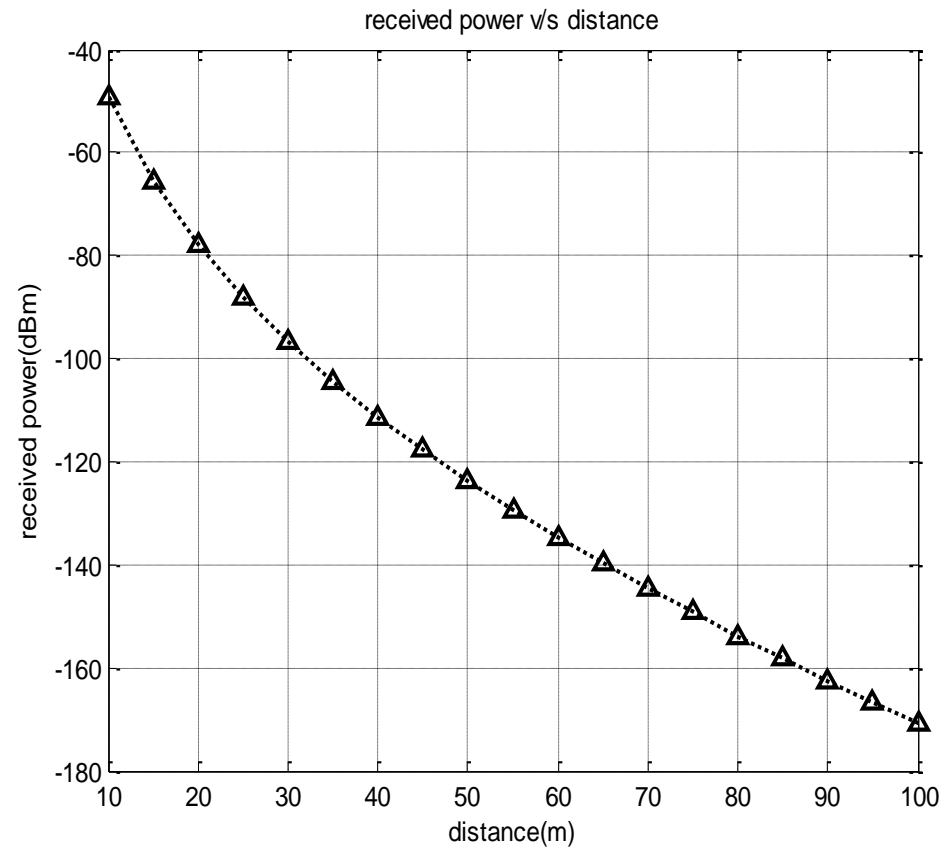

Fig 5.2 Received power Vs distance at 40m transmitter height.

The graph in the Fig 5.4 depicts the comparative variation of received power at varying distances at the transmitter heights $14 \mathrm{~m}, 40 \mathrm{~m}$ and $70 \mathrm{~m}$ and the other parameters taken are similar as in above result.

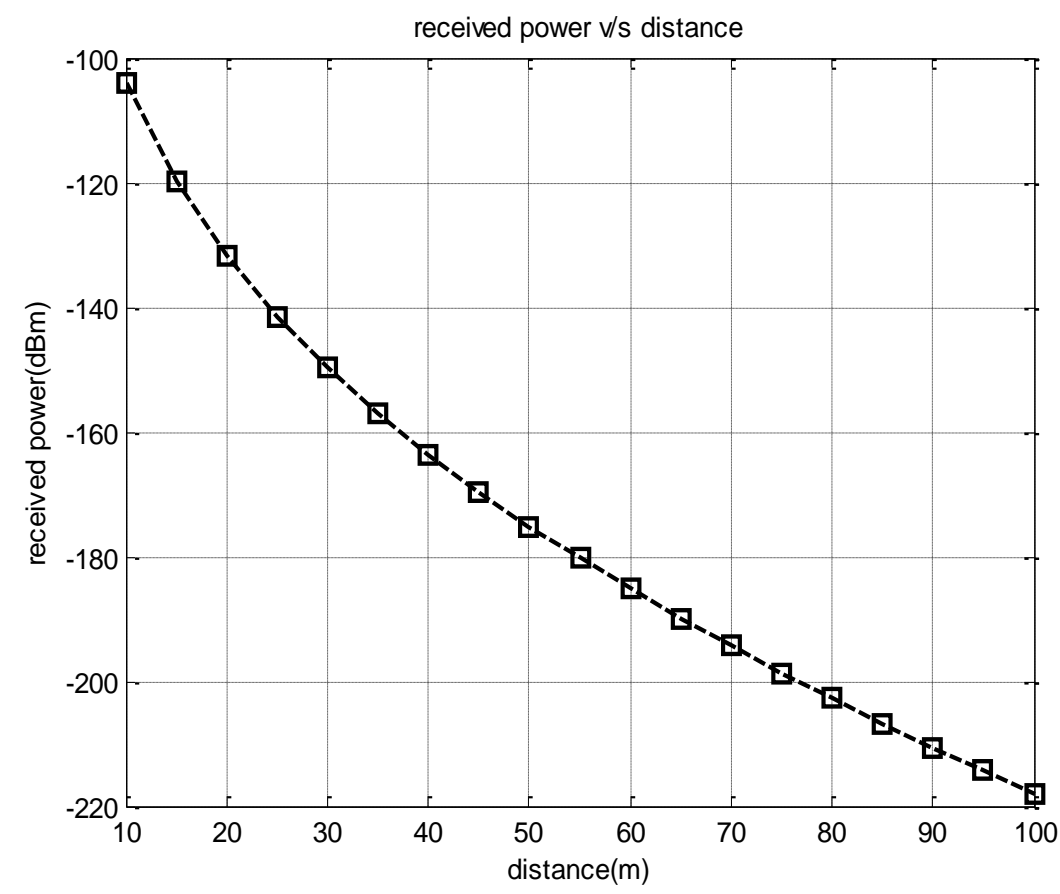

Fig 5.3 Received power Vs distance at 70m transmitter height 


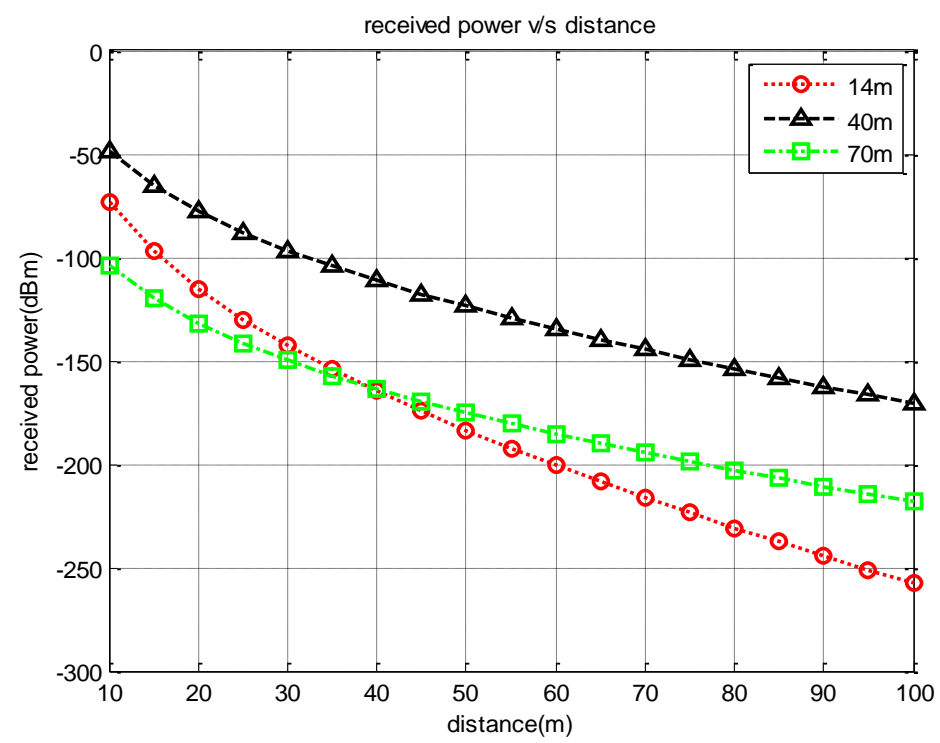

Fig. 5.4 Comparative analysis at transmitter heights $(14 \mathrm{~m}, 40 \mathrm{~m}$ and $70 \mathrm{~m})$

\subsection{TRANSMITTED POWER $\left(\mathbf{P}_{\mathrm{t}}\right)$ Vs DISTANCE $(\mathbf{d})$}

Normally sensors have initial battery power of 5Joule out of which the internally used energy by the internal electronics is 0.02Joule, remaining is used for transmission over the distances. With the increase in distance (d) between the nodes there is increase in the power requirement. Other external and internal parameters such as transmitted electronics energy $\left(e_{t}\right)$, amplifier consumption $\left(e_{d}\right)$, receiver electronics consumption energy $\left(\mathrm{e}_{\mathrm{r}}\right)$, bit rate $(\mathrm{B})$ and traffic $(\mathrm{A})$ play prominent role in determining transmitted power.

The graph in Fig 5.5 depicts the relationship between the transmitted power $\left(\mathrm{P}_{t}\right)$ at varying distance $(\mathrm{d})$ between the transmitting and receiving sensor nodes and the constant traffic for the different parameters of the values $\mathrm{e}_{\mathrm{t}}=50 * 10^{-9}$ Joule, $\mathrm{e}_{\mathrm{d}}=100 * 10^{-12}$ Joule, $\mathrm{A}=0.5, \mathrm{~B}=1 \mathrm{mbps}$ and $\mathrm{d}=55$.

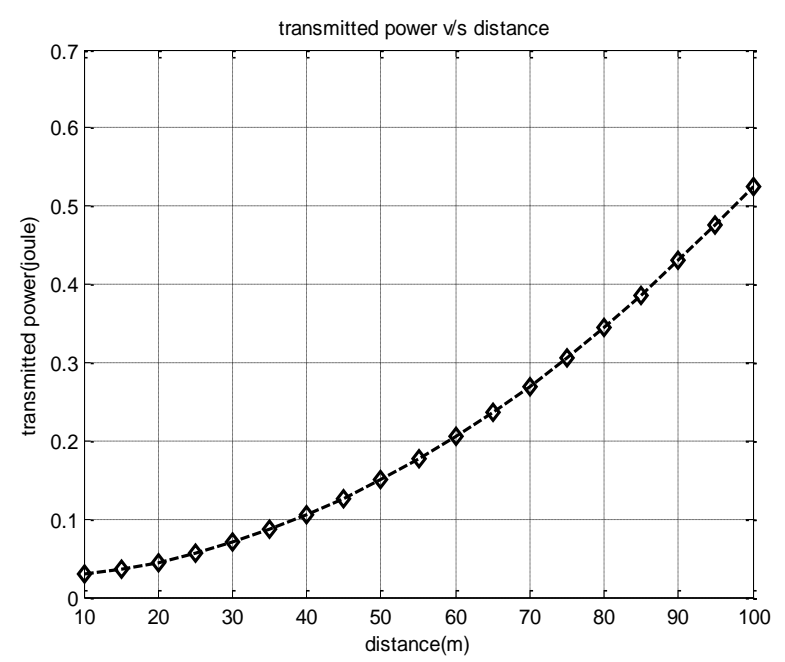

Fig. 5.5 Distance Vs Transmitted power at varying distances

\subsection{TRAFFIC Vs TRANSMITTED POWER $\left(\mathbf{P}_{\mathrm{t}}\right)$}

Traffic is the ratio of number of nodes communicating to the particular node to the total number of nodes. With the increase in the value of traffic the value of transmitted power required increases. In a WSN there are hundreds even thousands of sense nodes are deployed and each node can communicate with each other. Many times it happens that single node communicate with many nodes at a time, the traffic on that node increases many folds and the receiving capacity of that node decreases. More will be the traffic lesser will be the efficiency of accepting data from other nodes.

The value of traffic ranges from 0 to 1 . Suppose there are 100 nodes when no node is communicating with any node then the value of traffic is 0 and when 10 node are communicating at a time then to the single node then 
the traffic on that particular node will be $0.1(10 / 100)$ and so on, and at 100 nodes communicating or all nodes communicating the value will be maximum i.e. 1 .

For representing the relation, 20 nodes have been taken and traffic was calculated, and it was found that the transmitted power required increases linearly with the increase in the traffic, as shown in the Fig. 5.6.

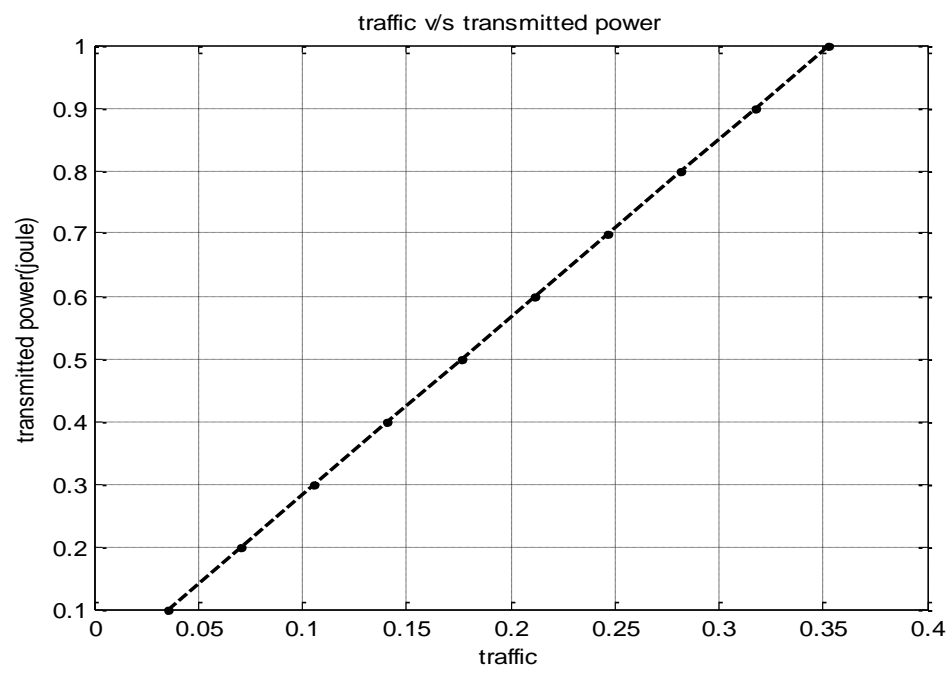

Fig 5.6 Traffic Vs Transmitted power at varying traffic

\section{CONCLUSION}

The results show the comparative analysis of received power with varying heights. It was observed that the received power of the sensor nodes decreases with increase in distance between transmitting and receiving nodes but it has different characteristics change at different transmitter heights as the path loss exponent varies with the heights.

The transmitted power requirement also varies with the changes in distances between the transmitting and receiving nodes, changing traffic etc. Transmitted power requirement increases exponentially with the distance while it is the linear variation of the increase in transmitted power requirement with the increase in the traffic.

\section{REFERENCES}

[1] Wireless sensor networks: a survey I.F. Akyildiz, W. Su*, Y. Sankarasubramaniam, E. Cayirci Broadband and Wireless Networking Laboratory, School of Electrical and Computer Engineering, Georgia Institute of Technology, Atlanta, GA 30332, USA Received 12 December 2001; accepted 20 December 2001.

[2] I. F. Akyilldiz, W. Su, Y. Sankarasubramaniam and E. Cayirci, (2002), "A survey on sensor networks," IEEE Communications Magazine, pp. 102-114.

[3] Pavlos Papageorgiou(July 16, 2003) "Literature Survey on Wireless Sensor Networks".

[4] Ashraf Hossain, P. K. Biswas, S. Chakrabarti“Sensing Models and Its Impact on Network Coverage in Wireless Sensor Network" 2008 IEEE Region 10 Colloquium and the Third ICIIS, Kharagpur, INDIA December 8-10.447.

[5] F. M. Al-Turjman, H. Hassanein, and M. Ibnkahla, "Connectivity optimization for wireless sensor networks applied to forest monitoring", In Proc. of the IEEE International Conf. on Communications (ICC), Dresden, 2009, pp. AHSN11.5.1-5.

[6] Giuseppe Anastasi*,Marco Conti\#, Mario Di Francesco*, Andrea Passarella\#," Energy Conservation in Wireless Sensor Networks".

[7] Q. Gao, K. J. Blow, D. J. Holding, I. W. Marshall , X. H. Peng "Radio Range Adjustment for Energy Efficient Wireless Sensor Networks", Electronic Engineering, Aston University, Birmingham B4 7ET,United Kingdom, Computing Laboratory, University of Kent, Canterbury, Kent CT2 7NZ, United Kingdom.

[8] T. Rappaport, Wireless Communications: Principles \& Practice, Prentice-Hall Inc., New Jersey, 1996.

[9] M. Bhardwaj, T. Garnett, A. P. Chandrakasan, Upper bounds on the lifetime of sensor networks, in: Proceedings of ICC'01, vol. 3, Helsinki, Finland, June 2001, pp. 785-790.

[10] J. Rodrigues, S. Fraiha, H. Gomes, G. Cavalcante, A. de Freitas, and G. de Carvalho, "Channel Propagation Model for Mobile Network Project in Densely Arboreous Environments," Journal of Microwaves and Optoelectronics, vol. 6, no. 1, p. 236, 2007. 
[11] Arman Alam Ansari", Mukesh Kumar*, Rohini Saxena*, Md. Saddam Hussain" " OPTIMIZATION MONITORING AND APPLICATION OF WIRELESS SENSOR NETWORKS TO FOREST: A SURVEY", SHIATS, Allahabad.

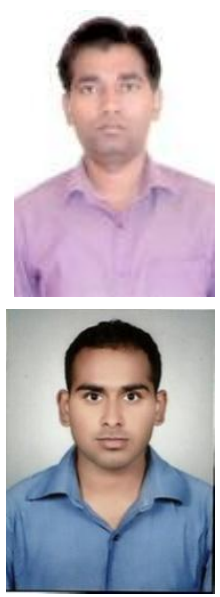

Mukesh Kumar is working as an Asst. Prof. in the Department of Electronics \& Communication Engineering in SHIATS, Allahabad. He received his M.Tech. Degree in Advanced Communication Systems Engineering from SHIATS, Allahabad in 2010. His research is focused on Wireless Sensors Networks and Computer Networks Microwave Engineering, as well as Optical fiber communication.

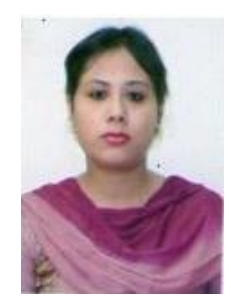

Rohini Saxena is working as a Asst. Prof. in the Department of Electronics \& Communication Engineering in SHIATS, Allahabad. She received her M.Tech. Degree in Advanced Communication Systems Engineering from SHIATS, Allahabad in 2009. His research is focused on Microwave Engineering, Wireless Sensors Networks and Computer Networks and Mobile communication.

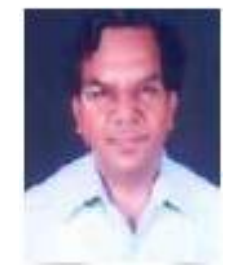

A.K. Jaiswal is working as professor and HOD in the department of ECE at SHIATS, Allahabad. He worked for the promotional activities in optical fibre communication system sponsored by Government of India, in association with IIT Kharagpur and Madras. His research interests are Optical system, Microwave, Sensors and Networks. 